\title{
Reliability of Emergency Department Diagnosis in Identifying the Etiology of Nontraumatic Undifferentiated Hypotension
}

\author{
Rameshbabu H Javali ${ }^{1}$, Arpitha Loganathan ${ }^{2}$, Madhu Srinivasarangan ${ }^{3}$, Akkamahadevi Patil ${ }^{4}$, Ganesha B Siddappa ${ }^{5}$, \\ Nisarg Satyanarayana ${ }^{6}$, Adarsh S Bheemanna $^{7}$, Sriharsha Jagadeesh ${ }^{8}$, Sagarika Betkerur ${ }^{9}$
}

\begin{abstract}
Introduction: Nontraumatic undifferentiated hypotension is one of the common and challenging critical presentations in the emergency department (ED) due to the difficulty in diagnosing the etiology of shock. In the present study, an attempt was made to test point-of-care ultrasound (PoCUS) as an early approach to improve the accuracy of diagnosis and to narrow the differentials in cases of nontraumatic undifferentiated hypotension.

Materials and methods: This is a prospective explorative study conducted in the ED of a tertiary care hospital over a period of 18 months. A total of 100 patients were included in the study. All patients $>18$ years of age with systolic blood pressure $<90 \mathrm{~mm} \mathrm{Hg}$ with at least one sign or symptom of hypoperfusion were included in the study. Patients referred from another hospital as shock, history of trauma, and history suggestive of orthostatic hypotension and presented with symptomatic postural hypotension as the only chief complaint were excluded. All the patients who met the inclusion/exclusion criteria underwent detailed clinical and multi-organ PoCUS evaluation by two different observers. Assessment of the lungs, cardia, abdomen, aorta, inferior vena cava (IVC), and leg veins during the PoCUS examination was done. A third observer combined the clinical evaluation and the PoCUS findings. All patients were followed through for their final diagnosis at the time of discharge. First, the diagnosis after clinical evaluation alone was compared to the final diagnosis. Then the diagnoses based on the findings of PoCUS alone were compared with the final diagnosis. Last, the diagnosis obtained on combining the data of clinical evaluation with that of PoCUS was compared to the final diagnosis. The data were analyzed based on their reliability indices, accuracy, and the Cohen's kappa coefficient.

Results: Diagnoses based on clinical evaluation alone and POCUS alone were found to be accurate in $45 \%$ and $47 \%$ of patients, respectively. But on combining the findings of clinical evaluation with PoCUS, the accuracy increased to $89 \%$. The most common etiology of shock was found to be distributive shock present in $38 \%$ of patients with sepsis being the most common subtype. In patients with obstructive shock, combined clinical evaluation with PoCUS was in perfect agreement with Cohen's kappa coefficient $(\kappa)=1$ and those with distributive shock were in substantial agreement with Cohen's kappa coefficient $(\kappa)=0717$. The overall kappa correlation of the combined evaluation with PoCUS was 0.89 , which shows an almost perfect agreement with the final diagnosis.

Conclusion: This study demonstrates the accuracy and reliability of PoCUS as an easy and valuable bedside tool when added to the clinical evaluation. It helps in narrowing the differentials and thereby guiding early goal-directed therapy in nontraumatic, undifferentiated hypotension patients presenting to the ED.
\end{abstract}

Keywords: Accuracy, Cardiogenic shock, Distributive shock, Emergency department, Emergency physician, Nontraumatic, Point-of-care ultrasound, Shock, Undifferentiated hypotension.

Indian Journal of Critical Care Medicine (2020): 10.5005/jp-journals-10071-23429

\section{INTRODUCTION}

In the practice of emergency medicine, the targeted and focused management of acutely ill medical patients is the cornerstone. Critically ill patients present a challenge to the emergency physician as they often present with etiological ambiguity, unavailable or unreliable history, or rapidly deteriorating and unstable clinical condition. ${ }^{1}$

Three previous studies have reported high mortality rates, $16-25 \%$, among emergency department (ED) patients with nontraumatic undifferentiated shock. ${ }^{2-4}$ These poor outcomes underline the importance of utilizing a systematic approach to determining the etiology of shock. ${ }^{1}$

Multi-organ ultrasound not only substantially decreases the practical delays associated with imaging and laboratory tests but can obviate the need for them when a definitive diagnosis is obtained on ultrasound imaging, avoiding a large radiation dose. ${ }^{5}$ It is the versality of ultrasound to rule in and exclude multiple different diagnoses that makes it an indispensable tool for an ED physician in identifying the etiology of undifferentiated shock.
${ }^{1-3,6-9}$ Department of Emergency Medicine, JSS Medical College, JSS Academy of Higher Education and Research, Mysuru, Karnataka, India

${ }^{4}$ Department of Anesthesia, JSS Medical College, JSS Academy of Higher Education and Research, Mysuru, Karnataka, India

${ }^{5}$ Department of Emergency Medicine, SSIMS\&RC, Davangere, Karnataka, India

Corresponding Author: Arpitha Loganathan, Department of Emergency Medicine, JSS Medical College, JSS Academy of Higher Education and Research, Mysuru, Karnataka, India, Phone: +91 9900688088, e-mail: arpi.loga@gmail.com

How to cite this article: Javali RH, Loganathan A, Srinivasarangan $M$ Akkamahadevi P, Ganesha BS, Nisarg S, et al. Reliability of Emergency Department Diagnosis in Identifying the Etiology of Nontraumatic Undifferentiated Hypotension. Indian J Crit Care Med 2020;24(5): 313-320.

Source of support: Nil

Conflict of interest: None

() The Author(s). 2020 Open Access This article is distributed under the terms of the Creative Commons Attribution 4.0 International License (https://creativecommons. org/licenses/by-nc/4.0/), which permits unrestricted use, distribution, and non-commercial reproduction in any medium, provided you give appropriate credit to the original author(s) and the source, provide a link to the Creative Commons license, and indicate if changes were made. The Creative Commons Public Domain Dedication waiver (http://creativecommons.org/publicdomain/zero/1.0/) applies to the data made available in this article, unless otherwise stated. 
The use of bedside ultrasound has been described in the evaluation of undifferentiated shock for well over two decades. ${ }^{6-10}$ Though many have described different techniques and protocols, few have looked at the reliability of this tool in the ED and its effect on narrowing the differentials.

Not many studies have been done in these parts of the country and the literature available is limited. Hence, an attempt was made to test multi-organ point-of-care ultrasound (PoCUS) as an early approach to improve the accuracy and to narrow the differential diagnoses, thus testing the effectiveness of guiding the emergency physician (EP) in early goal-directed therapy for better outcome in patients presenting with nontraumatic undifferentiated hypotension.

\section{Materials and Methods}

This is a single-center, prospective, explorative study performed over a period of 18 months in the Department of Emergency Medicine of a 1,800-bed teaching, tertiary-care hospital with over 50,000 ED visits per year. The subjects were enrolled based on convenience sampling as only one EP trained in POCUS did the evaluation. We included patients above the age of 18 years with systolic BP $<90 \mathrm{~mm} \mathrm{Hg}$ and shock index $>1$ (shock index = heart rate) systolic blood pressure) during the first evaluation and the presence of at least one of the following signs or symptoms of hypoperfusion unresponsiveness, altered mental status, syncope, respiratory distress, generalized fatigue, severe chest pain or abdominal pain. The exclusion criteria were as follows:

- History suggestive of orthostatic hypotension and presented with symptomatic postural hypotension as the only chief complaint

- History of low baseline blood pressure and not having any signs or symptoms related to hypotension

- Patients with a history of trauma in preceding 24 hours

- Discharged or transferred from another hospital after initial evaluation and treatment

The sample size was calculated based on the formula,

$$
n=z^{2} p q / z^{2}
$$

where, $n$-sample size; $z$-standard normal deviate set at 1.96 corresponding to $95 \%$ confidence level; $p$-proportion of study population undergoing POCUS; $q-1-p ; d-$ degree of accuracy set at 0.05 .

A total of 100 patients who met the criteria were included in the study. Approval was obtained from the institutional ethical committee (IEC). Informed written consents from patients, their legally authorized representatives, or close families were obtained and filed after thorough explanation of the study. The PoCUS exam was conducted parallel to the standard of care management of the patient, and its patient safety was approved by the clinical governance committee of the institution.

For evaluation of PoCUS, the portable ultrasound machine, SonoSite M-TURBO (FUJIFILM, Bothell, WA, USA), was used during the study. The phased transducer 5-1 MHz was used to view the heart. There were four major cardiac views considered, subcoastal view, parasternal long-axis view, parasternal short-axis view, and the apical four-chamber view, to examine the fluid collection in the pericardial sac, right ventricle (RV) diastolic collapse, left ventricle (LV) hypokinesia by visual examination of gross wall contraction and wall thickening or LV hyperkinesia, LV function, and signs of RV dilatation. The linear transducer $13-6 \mathrm{MHz}$ was used to view the lungs. Lung sliding sign, B profile, consolidation, air bronchograms, and pleural effusion were recorded. The curved transducer 5-2 $\mathrm{MHz}$ was used to examine the free fluid in the hepatorenal pouch, the splenorenal pouch, and the pelvic cavity. The curved transducer 5-2 $\mathrm{MHz}$ was also used to assess the aorta for features of aneurysm and occlusion and inferior vena cava (IVC) collapsibility. Maximum diameter of $2 \mathrm{~cm}$ and $50 \%$ collapsibility was considered as the cutoff. The linear transducer $13-6 \mathrm{MHz}$ was used to examine the common femoral and popliteal leg veins for collapsibility and presence of intraluminal thrombosis.

\section{Method of Collection of Data}

Patients satisfying the inclusion criteria were interviewed and demographic data such as age, sex, and presenting complaints were noted. The diagnoses for this study were recorded as a type of or as a combination of types of shock.

Patients were subjected to thorough clinical examination and evaluated for vital signs, physical examination, and detailed secondary survey by one observer and the diagnosis was declared as Diagnosis 1. These patients were then subjected to a multi-organ PoCUS in the ED by a trained EP blinded to the clinical evaluation. The ultrasound findings were recorded on a predesigned proforma and a diagnosis based only on these ultrasound findings was declared as Diagnosis 2. The findings of the detailed clinical evaluation were combined with the findings of multi-organ PoCUS and a third diagnosis was declared based on the combined findings (Diagnosis 3). This was done by a different third observer, also an EP. All patients were followed during their hospitalization in order to document their final diagnosis. Patients were transferred to other medical/surgical units (internal medicine, cardiology, or surgery) and the final clinical diagnosis (Diagnosis 4) was established by the treating physician in charge. The treating physicians were certified specialists with acceptable expertise in their fields. These physicians were blind to the diagnoses declared in the patients' data sheet in the ED. Specific criteria were followed for establishing the diagnosis during the study (Annexures 1 to 3 ).

\section{Statistical Analysis}

The data were entered into MS Excel followed by the analysis using SPSS (v.22), licensed to JSS Academy of Higher Education and Research. Arithmetic mean, standard deviation, and percentages were used to represent age and gender distribution, vitals, etc.

The accuracy of diagnosis after clinical evaluation alone vs final diagnosis (Diagnosis 1 vs Diagnosis 4), diagnosis based on PoCUS alone vs final diagnosis (Diagnosis 2 vs Diagnosis 4), and diagnosis after combined clinical evaluation and PoCUS vs final diagnosis (Diagnosis 3 vs Diagnosis 4) was analyzed.

The sensitivity, specificity, positive predictive value, negative predictive value, and accuracy were calculated separately for Diagnosis 1, Diagnosis 2, and Diagnosis 3. The agreement between diagnosis 3 and the final diagnosis has been compared using Cohen's kappa coefficient $(\kappa)$.

\section{Results}

In the present study, it was observed that the mean age group of patients presenting with undifferentiated hypotension was $51.7 \pm$ 18.88 with a male predominance of $60 \%$. The mean systolic blood pressure (SBP) and diastolic blood pressures (DBP) were $78.5 \pm$ 
10.57 and $35.8 \pm 27.67$ in this study population. The mean pulse rate and respiratory rate of patients in this study group was found to be 106.98 and 28.5 , respectively.

In this study, by clinical evaluation alone (Diagnosis 1), $45 \%$ of patients were diagnosed accurately when compared to the final diagnosis (Diagnosis 4).

About $47 \%$ of patients were diagnosed accurately on PoCUS alone (Diagnosis 2) when compared to the final diagnosis (Diagnosis 4).

On combining the clinical evaluation with PoCUS (Diagnosis 3), $89 \%$ of patients matched accurately to the final diagnosis (Diagnosis 4) as described in Figure 1.

The subgroup analysis of individual types of shock was then attempted. The most common diagnosis among this study group was distributive shock after combined clinical evaluation with PoCUS and final diagnosis.

It was observed that in patients diagnosed with cardiogenic shock, clinical evaluation alone was more specific (91\%) and PoCUS alone was more sensitive (100\%), but on combining PoCUS with

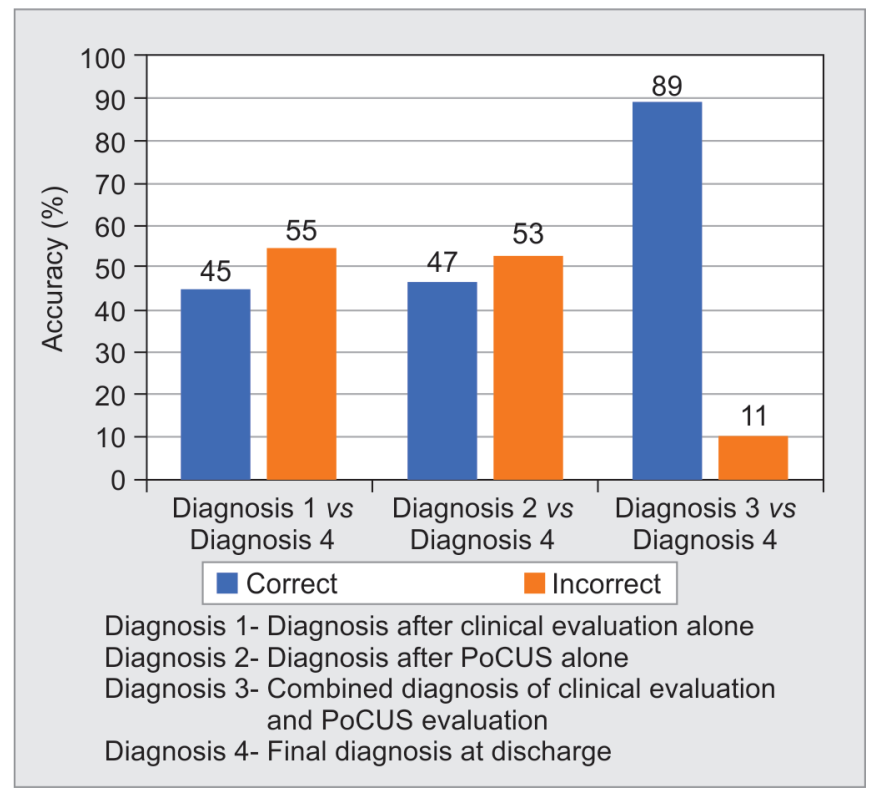

Fig. 1: The statistical diagnostic accuracy of patients $(n=100)$ clinical evaluation, the reliability rose to $>98 \%$ with an accuracy of $\kappa=0.95$, which indicates almost perfect agreement with the final diagnosis. It was also found that combining clinical evaluation with PoCUS (Diagnosis 3) was very efficient in ruling out cardiogenic shock as a probable etiology in patients with unknown shock $(\mathrm{NPV}=100 \%)$. Similar findings were also found in patients with hypovolemic shock.

In case of patients diagnosed with obstructive and distributive shock both clinical evaluation alone (Diagnosis 1) and PoCUS alone (Diagnosis 2 ) were highly specific ( 100\%). On combining the clinical evaluation and PoCUS, it was found that in patients with obstructive shock Diagnosis 3 was in perfect agreement with final diagnosis (Diagnosis 4) $(\kappa=1)$. Patients with distributive shock had $\kappa=0.717$ (substantial agreement) with Diagnosis 3 when compared to the final diagnosis.

The diagnosis of patients with mixed type of shock was not highly specific or sensitive when clinical evaluation alone or PoCUS alone was used, but on adding the PoCUS findings to the clinical evaluation, the sensitivity and specificity rose to $>90 \%$ and the accuracy of Diagnosis 3 was in substantial agreement with that of the final diagnosis $(\kappa=0.76)$ as described in Tables 1 to 3 and Figure 2 .

\section{Discussion}

Shock can be an elusive diagnosis. There is no single clinical parameter or diagnostic study that can accurately predict the presence of circulatory insufficiency. The ability to correctly assess the etiology of shock has been described as the essence of the discipline of emergency medicine. ${ }^{3,11}$ This study adds to the evidence that a goal-directed point-of-care ultrasound will help EPs correctly identify the cause of nontraumatic symptomatic undifferentiated hypotension. ${ }^{3,12}$ It further demonstrates that PoCUS when added to the standard clinical evaluation can benefit the EP by narrowing the differential diagnoses in such patients (Fig. 3).

In the present study conducted on 100 patients presenting with undifferentiated hypotension as per the inclusion and exclusion criteria, the diagnosis at admission, after detailed clinical evaluation alone, the diagnosis after PoCUS alone, and the combined diagnosis of clinical evaluation and PoCUS were compared with that of the diagnosis at the time of discharge after complete evaluation.

Table 1: Reliability indices of Diagnosis 1 (clinical evaluation alone) vs Diagnosis 4 (final diagnosis) of patients

\begin{tabular}{lllcll}
\hline & $\begin{array}{l}\text { Cardiogenic shock } \\
(n=21)(\%)\end{array}$ & $\begin{array}{l}\text { Hypovolemic shock } \\
(n=8)(\%)\end{array}$ & $\begin{array}{l}\text { Obstructive shock } \\
(n=2)(\%)\end{array}$ & $\begin{array}{l}\text { Distributive shock } \\
(n=12)(\%)\end{array}$ & $\begin{array}{l}\text { Mixed shock ( } n=39) \\
(\%)\end{array}$ \\
\hline Sensitivity & 70.00 & 41.00 & 50.00 & 28.00 & 44.00 \\
Specificity & 91.00 & 96.50 & 100.00 & 98.00 & 63.50 \\
PPV & 66.00 & 62.50 & 100.00 & 75.00 & 28.20 \\
NPV & 92.00 & 92.30 & 97.00 & 73.80 & 77.00 \\
\hline
\end{tabular}

Table 2: Reliability indices of Diagnosis 2 (PoCUS evaluation alone) vs Diagnosis 4 (final diagnosis) of patients

\begin{tabular}{lllccc}
\hline & $\begin{array}{l}\text { Cardiogenic shock } \\
(n=27)(\%)\end{array}$ & $\begin{array}{l}\text { Hypovolemic shock } \\
(n=34)(\%)\end{array}$ & $\begin{array}{l}\text { Obstructive shock } \\
(n=3)(\%)\end{array}$ & $\begin{array}{l}\text { Distributive shock } \\
(n=5)(\%)\end{array}$ & Mixed $(n=27)(\%)$ \\
\hline Sensitivity & 100.00 & 83.00 & 75.00 & 15.00 & 30.70 \\
Specificity & 90.10 & 72.70 & 100.00 & 100.00 & 74.30 \\
PPV & 70.30 & 29.40 & 100.00 & 100.00 & 29.00 \\
NPV & 100.00 & 96.90 & 98.00 & 71.50 & 75.30 \\
\hline
\end{tabular}


Table 3: Reliability indices and $\kappa$ agreement of Diagnosis 3 (combined clinical and PoCUS evaluation) vs Diagnosis 4 (final diagnosis) for each type of shock in patients

\begin{tabular}{lccccc}
\hline & $\begin{array}{l}\text { Cardiogenic shock } \\
(n=20)(\%)\end{array}$ & $\begin{array}{l}\text { Hypovolemic shock } \\
(n=16)(\%)\end{array}$ & $\begin{array}{l}\text { Obstructive shock } \\
(n=4)(\%)\end{array}$ & $\begin{array}{l}\text { Distributive shock } \\
(n=32)(\%)\end{array}$ & $\begin{array}{l}\text { Mixed shock ( } n=28) \\
(\%)\end{array}$ \\
\hline Sensitivity & 100.00 & 100.00 & 100.00 & 73.68 & 92.00 \\
Specificity & 98.70 & 98.00 & 100.00 & 100.00 & 90.41 \\
PPV & 95.20 & 85.70 & 100.00 & 100.00 & 76.47 \\
NPV & 100.00 & 100.00 & 100.00 & 86.11 & 97.00 \\
Cohen's kappa & 0.9500 & 0.8570 & 1 & 0.7170 & 0.7640 \\
coefficient $(\kappa)$ & & & & & \\
\hline
\end{tabular}

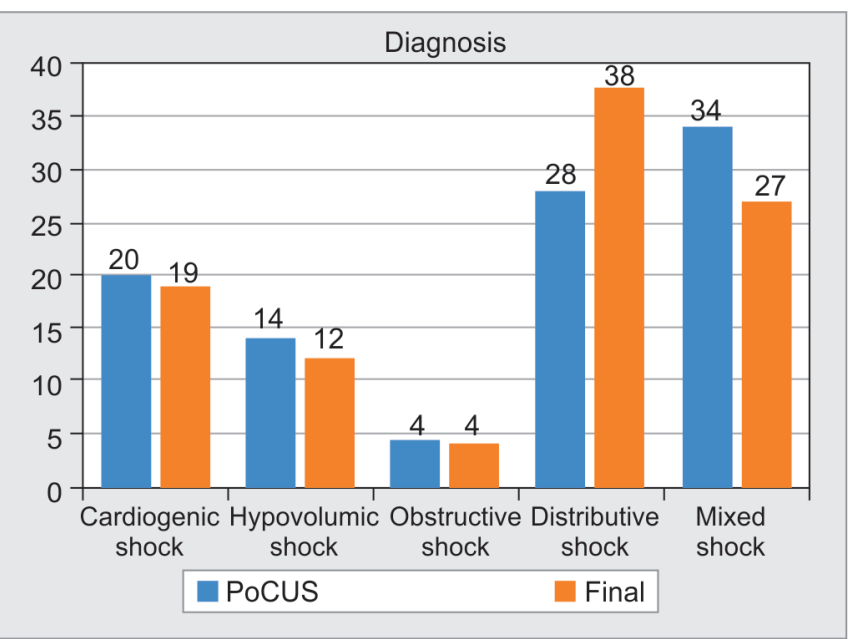

Fig. 2: Combined diagnosis of clinical evaluation with PoCUS (Diagnosis 3 ) and final diagnosis (Diagnosis 4$)$ of patients $(n=100)$

The demographic profile was comparable to a study conducted by Atkinson et al. ${ }^{7}$ The vitals and clinical characteristics are comparable with studies done by Jones et al. and Shokoohi et al., which showed similar results. These have been illustrated in the Table 4. ${ }^{2,13}$

Among the study group, most patients (38\%) were diagnosed to have distributive type of shock. Of these, sepsis was the commonest subtype, found in $84 \%$ of patients. This was a similar finding in other studies as well. ${ }^{6,13-17}$ In most cases, distributive shock was missed in Diagnosis 2; this may be so because classifying a patient as distributive shock based on PoCUS alone is difficult except in case of sepsis where foci of sepsis, such as consolidation, air bronchogram, gallbladder wall thickening, limb cellulitis, or hypoechoic pancreas, is present. In all other causes of distributive shock, associated hypovolemia will be the only finding on PoCUS alone. In these cases, the diagnosis would be recorded as hypovolemic shock. Hyperkinetic LV was a common finding in patients with distributive shock. The presence of hyperkinetic LV in PoCUS as an independent predictor of septic shock is illustrated by a study done by Jones et al. ${ }^{1,13}$

Obstructive shock was found to be difficult to diagnose with clinical evaluation alone. However, with high clinical suspicion, addition of PoCUS was very efficient in picking up the diagnosis and also equally reliable in excluding the diagnosis of obstructive shock. This is especially important in obstructive shock as the management in these cases is specific and time is of the essence.

The trained EP's determination of EF using subjective measurement was found to be comparable to that of the cardiologist's estimate of EF in $88 \%$ of patients with hypokinesia.

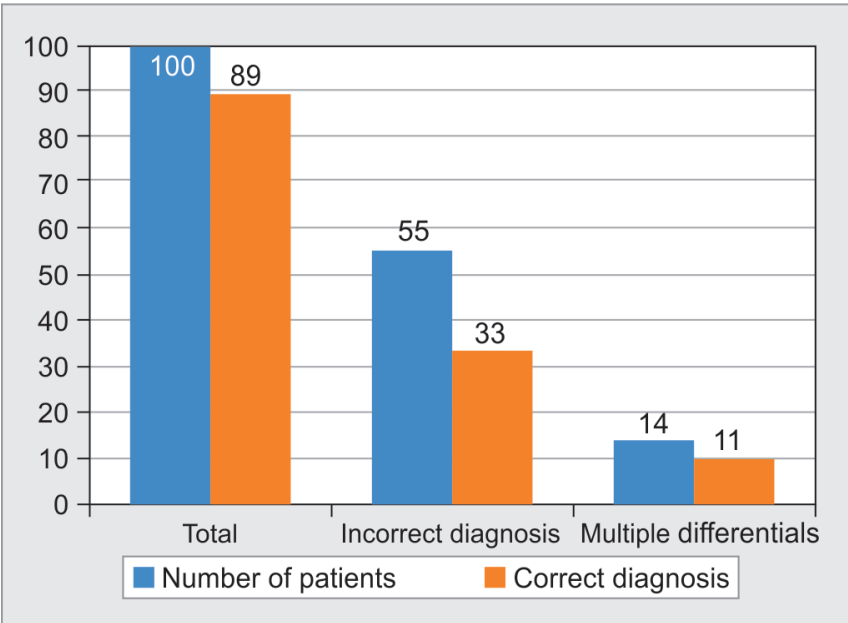

Fig. 3: Number of patients with corrected diagnosis on adding PoCUS finding to the clinical diagnosis (Diagnosis 1)

This finding was similar to two other studies by Mueller, et al., and Moore et al. ${ }^{4,18}$ The overestimate of hypokinetic LV after PoCUS was found in patients with mixed shock of distributive and cardiogenic shock, where the cardiogenic component may have resolved due to early treatment, which would have normalized on subsequent scan.

All three combinations of shock (cardiogenic with obstructive, cardiogenic with distributive, and hypovolemic with distributive) were grouped as mixed shock for convenience and for comparison. Our study was comparable to three other studies, Ghane et al. and Bagheri et al. based on the RUSH protocol and Ahn et al. based on the SEARCH8E protocol, $6,15,17$ as illustrated in Table 5.

The accuracy of ED diagnosis was found to be in almost perfect agreement with the final diagnosis with Cohen's kappa coefficient of 0.89 . This was similar to that of Ahn et al., Volpicelli et al., and Shokoohi et al. study among other studies. 6,15,17,19,20

The observations based on our study data reinforce the importance of developing a specific diagnostic algorithm to elucidate the etiology of undifferentiated hypotension as majority of the patients warranted specific and unique treatments. In a way, there might be a window of opportunity for the EP to provide more accurate diagnosis and a more specific treatment in this set of patients than just infusion of crystalloid and monitoring of hemodynamic indices. ${ }^{2,3}$ Access to the PoCUS information allowed EPs to be almost $40 \%$ more accurate than clinicians who had access to information on standard clinical examination alone. As with any imaging study, the findings of the PoCUS need to be interpreted in the context of the clinical setting. This helps explain why abnormal findings on PoCUS alone sometimes did not match the final diagnosis and often required further interpretation to 
Table 4: Comparison of demographic profile, vitals, and clinical characteristics of patients

\begin{tabular}{llll}
\hline Variable & Present study & $\begin{array}{l}\text { Jones et al. study }{ }^{2} \\
\text { (adverse outcome) }\end{array}$ & $\begin{array}{l}\text { Shokoohi et al. } \\
\text { study }\end{array}$ \\
\hline Age (years) & $51.7 \pm 18.88$ & $58 \pm 15.6$ & 61.6 \\
Gender (\%) & M-60 & M-80.2 & M-61.0 \\
SBP (mm Hg) & 78.5 & $83 \pm 12.8$ & 74.6 \\
DBP (mm Hg) & 35.8 & - & 44.8 \\
Pulse rate (beats/minute) & 106.98 & $108 \pm 28.5$ & 94.9 \\
Respiratory rate (breaths/minute) & 28.5 & $26 \pm 8.1$ & - \\
Oxygen saturation (\%) & 85.99 & $93 \pm 7.7$ & - \\
GRBS (gm/dL) & $176.85 \pm 113.26$ & $161 \pm 97.4$ & \\
Temperature ( $\left.{ }^{\circ} \mathrm{F}\right)$ & $99.22 \pm 1.89$ & $99 \pm 2.8$ & 98.0 \\
Total WBC count (cells/mm ${ }^{3}$ ) & $16.30 \pm 7.39$ & $12 \pm 6.6$ & 9.26 \\
Comorbidities & Hypertension (39\%), & & Hypertension (56\%), congestive \\
& diabetes (33\%) & & heart failure (33\%) \\
\hline
\end{tabular}

Table 5: Comparison of reliability indices and Cohen's kappa coefficient

\begin{tabular}{|c|c|c|c|c|c|}
\hline Type of shock & Reliability indices & Our study $(n=100)$ & Ghane et al. $(n=77)$ & $\begin{array}{l}\text { Bagheri-Hariri et al. } \\
(n=148)\end{array}$ & Ahn et al. $(n=308)$ \\
\hline \multirow[t]{5}{*}{ Cardiogenic } & Sensitivity (\%) & 100.00 & 90.00 & 60.00 & 94.30 \\
\hline & Specificity (\%) & 98.70 & 98.00 & 100.00 & 97.90 \\
\hline & PPV (\%) & 95.20 & 94.70 & 100.00 & 93.00 \\
\hline & NPV (\%) & 100.00 & 97.00 & 90.9 & 98.30 \\
\hline & Cohen's $(\kappa)$ & 0.9500 & 0.89 & 0.71 & 0.90 \\
\hline \multirow[t]{5}{*}{ Hypovolemic } & Sensitivity (\%) & 100.00 & 100.00 & 100.00 & 100.00 \\
\hline & Specificity (\%) & 98.00 & 96.20 & 72.7 & 98.40 \\
\hline & PPV (\%) & 85.70 & 88.90 & 82.4 & 28.60 \\
\hline & NPV (\%) & 100.00 & 100.00 & 100.00 & 100.00 \\
\hline & Cohen's $(\kappa)$ & 0.8570 & 0.920 & 0.750 & 0.439 \\
\hline \multirow[t]{5}{*}{ Obstructive } & Sensitivity (\%) & 100.00 & 90.90 & - & 100.00 \\
\hline & Specificity (\%) & 100.00 & 98.20 & & 100.00 \\
\hline & PPV (\%) & 100.00 & 90.90 & & 100.00 \\
\hline & NPV (\%) & 100.00 & 98.30 & & 100.00 \\
\hline & Cohen's $(\kappa)$ & 1.0000 & 0.890 & & 1.00 \\
\hline \multirow[t]{5}{*}{ Distributive } & Sensitivity (\%) & 73.68 & 72.70 & 75.00 & 63.60 \\
\hline & Specificity (\%) & 100.00 & 100.00 & 100.00 & 99.70 \\
\hline & PPV (\%) & 100.00 & 100.00 & 100.00 & 87.50 \\
\hline & NPV (\%) & 86.11 & 95.10 & 95.50 & 98.70 \\
\hline & Cohen's $(\kappa)$ & 0.7170 & 0.810 & 0.830 & 0.729 \\
\hline \multirow[t]{5}{*}{ Mixed } & Sensitivity (\%) & 92.00 & 63.6 & 100.00 & - \\
\hline & Specificity (\%) & 90.41 & 98.2 & 100.00 & \\
\hline & PPV (\%) & 76.47 & 87.5 & 100.00 & \\
\hline & NPV (\%) & 97.00 & 93.3 & 100.00 & \\
\hline & Cohen's $(\kappa)$ & 0.7640 & 0.700 & 1.000 & \\
\hline Composite & Cohen's $(\kappa)$ & 0.89 & 0.71 & 0.84 & 0.870 \\
\hline
\end{tabular}

determine whether the findings were significant. For example, $36 \%$ of patients had severe cardiac dysfunction, but the EPs needed to use data from clinical examination to decide if this finding indicated a significant pathological process (e.g., myocardial ischemia or hypodynamic sepsis) or a process that was probably not the cause of hypotension (e.g., chronic heart failure in a dehydrated patient). Indeed, the data suggest that normal findings on ultrasound examination were often used by EPs to objectively exclude potential causes of undifferentiated hypotension that are otherwise difficult to eliminate at the bedside (e.g., abdominal aortic aneurysm).
The addition of the PoCUS to standard care afforded EPs with the ability to compile a more accurate list of possible causes of nontraumatic undifferentiated hypotension. Narrowing the differentials drawn at clinical evaluation alone to a single diagnosis is especially important in patients with diagnosis of obstructive and cardiogenic shock, where even small delay in diagnosis and treatment can result in fatality. Looking at the subgroup of those patients with multiple differentials after clinical evaluation $(n=$ 14), the addition of PoCUS gave a single true diagnosis in $79 \%$ ( $n=$ 11). In 44 out of 55 patients, where the admission diagnoses based 
on clinical evaluation alone could have been incorrect, addition of data from multi-organ PoCUS resulted in a change to the correct diagnoses.

This suggests that while clinical evaluation alone or PoCUS alone is equally unreliable in precisely diagnosing a patient with unknown shock, the use of PoCUS as a supplement to clinical evaluation markedly improves accuracy of diagnosis in the ED itself and can guide appropriate treatment.

\section{Conclusion}

This study contributes to the body of evidence supporting the use of multi-organ PoCUS in patients presenting with undifferentiated hypotension to the ED. Clinical evaluation alone was found to be poorly sensitive or specific in the critically ill and rapidly deteriorating patients. Supplementation with focused PoCUS allowed the diagnostic accuracy to rise to reliable levels. It has proved to be a valuable tool in narrowing the possible differentials, thereby guiding the EP in an early goal-directed therapy and improving the final outcome of the patients. This combined clinical evaluation and PoCUS can be used as a routine approach and should form part of the standard training for all EPs dealing with this patient group.

\section{Limitation}

This was a single-center study. The study group was limited to a small number of 100 patients.

- We have considered only consolidation and air bronchogram as foci of sepsis.

- No systematic method was used to assign the patients for PoCUS evaluation.

- This study did not incorporate any goal-directed outcome like time to definitive treatment or in-hospital mortality.

\section{ACKnowledgments}

We would like to acknowledge the generous contribution toward the conduct of this study by our colleagues, Dr Veeresh Balkal, Dr Ruben Omar, Dr Abhijith Sivashankar, Dr Suraj GB, Dr Krishnamoorthy DGSR, Dr Aishwarya Krishnan, and Dr Srivatsa S, our nursing staff, and the participating patients.

\section{References}

1. Mosier JM, Stolz U, Milligan R, Roy-Chaudhury A, Lutrick K, Hypes $C D$, et al. Impact of point-of-care ultrasound in the emergency department on care processes and outcomes in critically ill nontraumatic patients. Crit Care Explor 2019;1(6):e0019. DOI: 10.1097/ CCE.0000000000000019.

2. Jones $A E$, Aborn LS, Kline JA. Severity of emergency department hypotension predicts adverse hospital outcome. Shock 2004;22(5):410-414. DOI: 10.1097/01.shk.0000142186.95718.82.

3. Jones AE, Tayal VS, Sullivan DM, Kline JA. Randomized, controlled trial of immediate versus delayed goal-directed ultrasound to identify the cause of nontraumatic hypotension in emergency department patients. Crit Care Med 2004;32(8):1703-1708. DOI: 10.1097/01. ccm.0000133017.34137.82.

4. Moore CL. Determination of left Ventricular function by emergency physician echocardiography of hypotensive patients. Acad Emerg Med 2002;9(3):186-193. DOI: 10.1197/aemj.9.3.186.

5. Holler JG, Bech CN, Henriksen DP, Mikkelsen S, Pedersen C, Lassen AT. Nontraumatic hypotension and shock in the emergency department and the prehospital setting, prevalence, etiology, and mortality: a systematic review. Calvert J, ed. PLOS ONE 2015;10(3):e0119331. DOI: 10.1371/journal.pone.0119331.

6. Ahn JH, Jeon J, Toh H-C, Noble VE, Kim JS, Kim YS, et al. Search 8Es: a novel point of care ultrasound protocol for patients with chest pain, dyspnea or symptomatic hypotension in the emergency department. Brakenridge S, ed. PLOS ONE 2017;12(3):e0174581. DOI: 10.1371/ journal.pone.0174581.

7. Atkinson PRT, McAuley DJ, Kendall RJ, Abeyakoon O, Reid CG, Connolly J, et al. Abdominal and cardiac evaluation with sonography in shock (ACES): an approach by emergency physicians for the use of ultrasound in patients with undifferentiated hypotension. Emerg Med J 2009;26(2):87-91. DOI: 10.1136/emj.2007.056242.

8. Rose JS, Bair AE, Mandavia D, Kinser DJ. The UHP ultrasound protocol: a novel ultrasound approach to the empiric evaluation of the undifferentiated hypotensive patient. Am J Emerg Med 2001;19(4):299-302. DOI: 10.1053/ajem.2001.24481.

9. Lichtenstein DA, Mezière GA. Relevance of lung ultrasound in the diagnosis of acute respiratory failure*: the BLUE protocol. Chest 2008;134(1):117-125. DOI: 10.1378/chest.07-2800.

10. Perera $P$, Mailhot T, Riley $D$, Mandavia $D$. The RUSH exam: rapid ultrasound in shock in the evaluation of the critically ill. Emerg Med Clin North Am 2010;28(1):29-56. DOI: 10.1016/j.emc.2009.09.010.

11. Rosen's Emergency Medicine: Concepts and Clinical Practice - 9th Edition [Internet]. [cited 2019 Dec 28]. Available from: https://www. elsevier.com/books/rosens-emergency-medicine-concepts-andclinical-practice/walls/978-0-323-35479-0.

12. Sasmaz MI, Gungor F, Guven R, Akyol KC, Kozaci N, Kesapli M. Effect of focused bedside ultrasonography in hypotensive patients on the clinical decision of emergency physicians. Emerg Med Int 2017;2017:1-8. DOI: 10.1155/2017/6248687.

13. Shokoohi H, Boniface KS, Pourmand A, Liu YT, Davison DL, Hawkins KD, et al. Bedside ultrasound reduces diagnostic uncertainty and guides resuscitation in patients with undifferentiated hypotension. Crit Care Med 2015;43(12):2562-2569. DOI: 10.1097/CCM.0000000000001285.

14. Haydar SA, Moore ET, Higgins GL, Irish CB, Owens WB, Strout TD. Effect of bedside ultrasonography on the certainty of physician clinical decision making for septic patients in the emergency department. Ann Emerg Med 2012;60(3):346-358. DOI: 10.1016/ j.annemergmed.2012.01.006.e4.

15. Bagheri-Hariri S, Yekesadat M, Farahmand S, Arbab M, Sedaghat M, Shahlafar N, et al. The impact of using RUSH protocol for diagnosing the type of unknown shock in the emergency department. Emerg Radiol 2015;22(5):517-520. DOI: 10.1007/s10140-015-1311-z.

16. Jones AE, CraddockPA, Tayal VS. Diagnostic accuracy of left ventricular function for identifying sepsis among emergency department patients with nontraumatic symptomatic undifferentiated hypotension. Shock 2005;24(6):513-517. DOI: 10.1097/01.shk.0000186931.02852.5f.

17. Ghane MR, Gharib M, Ebrahimi A, Saeedi M, Akbari-Kamrani M, Rezaee M, et al. Accuracy of early rapid ultrasound in shock (RUSH) examination performed by emergency physician for diagnosis of shock etiology in critically ill patients. J Emerg Trauma Shock 2015;8(1):5. DOI: 10.4103/0974-2700.145406.

18. Mueller X, Stauffer JC, Jaussi A, Goy JJ, Kappenberger L. Subjective visual echocardiographic estimate of left ventricular ejection fraction as an alternative to conventional echocardiographic methods: comparison with contrast angiography. Clin Cardiol 1991;14(11): 898-902. DOI: 10.1002/clc.4960141108.

19. Shokoohi H, Boniface KS, Zaragoza M, Pourmand A, Earls JP. Point-of-care ultrasound leads to diagnostic shifts in patients with undifferentiated hypotension. Am J Emerg Med 2017;35(12):1984. e3-1984.e7. DOI: 10.1016/j.ajem.2017.08.054.

20. Volpicelli G, Lamorte A, Tullio M, Cardinale L, Giraudo M, Stefanone V, et al. Point-of-care multiorgan ultrasonography for the evaluation of undifferentiated hypotension in the emergency department. Intensive Care Med 2013;39(7):1290-1298. DOI: 10.1007/s00134-013-2919-7. 
Annexure 1: Criteria for establishing Diagnosis 1 (clinical evaluation alone)

\begin{tabular}{|c|c|}
\hline \multirow[t]{6}{*}{ Cardiogenic shock } & $\begin{array}{l}\text { Any or a combination of the below } \\
\text { associated with chest pain }\end{array}$ \\
\hline & $\begin{array}{l}\text { - Syncope, pain abdomen, generalized } \\
\text { weakness, seizures, altered sensorium, } \\
\text { vomiting, loose stools }\end{array}$ \\
\hline & $\begin{array}{l}\text { - ECG findings with ST elevation, ST } \\
\text { depression, T inversion, LBBB }\end{array}$ \\
\hline & $\begin{array}{l}\text { Raised JVP, pedal edema, anasarca, } \\
\text { ascites, tender hepatomegaly, } \\
\text { hepatojugular reflex }\end{array}$ \\
\hline & $\begin{array}{l}\text { History of progressive breathlessness, } \\
\text { paroxysmal nocturnal dyspnea, } \\
\text { orthopnea, dyspnea on exertion }\end{array}$ \\
\hline & $\begin{array}{l}\text { Past history of cardiac, pulmonary, or } \\
\text { renal disease, diabetes, hypertension }\end{array}$ \\
\hline \multirow[t]{4}{*}{ Hypovolemic shock } & $\begin{array}{l}\text { Any or a combination of the below } \\
\text { associated with vomiting or loose } \\
\text { stools or both without fever and } \\
\text { normal temperature at ED evaluation }\end{array}$ \\
\hline & - Pain abdomen, generalized weakness \\
\hline & $\begin{array}{l}\text { History of hematemesis, bleeding per } \\
\text { rectum, hemoptysis, heat stoke }\end{array}$ \\
\hline & $\begin{array}{l}\text { - UPT positive with sudden onset of } \\
\text { abdominal pain }\end{array}$ \\
\hline Obstructive shock & $\begin{array}{l}\text { - Sudden-onset breathlessness or chest } \\
\text { pain with history suggestive of DVT }\end{array}$ \\
\hline \multirow[t]{5}{*}{ Distributive shock } & $\begin{array}{l}\text { - Any or combination of the below with } \\
\text { fever or temperature }>100.4^{\circ} \mathrm{F} \text { at ED } \\
\text { evaluation }\end{array}$ \\
\hline & $\begin{array}{l}\text { Altered sensorium, breathlessness, } \\
\text { pain abdomen, generalized weakness, } \\
\text { cough, seizures, vomiting, loose stools }\end{array}$ \\
\hline & $\begin{array}{l}\text { - Only fever or temperature }>100.4^{\circ} \mathrm{F} \text { at } \\
\text { ED evaluation }\end{array}$ \\
\hline & $\begin{array}{l}\text { Evident focus of sepsis on clinical } \\
\text { examination }\end{array}$ \\
\hline & $\begin{array}{l}\text { History suggestive of anaphylaxis, } \\
\text { hepatic insufficiency, pancreatic } \\
\text { insufficiency, CVA, nontraumatic SAH, } \\
\text { spinal cord pathology }\end{array}$ \\
\hline $\begin{array}{l}\text { Cardiogenic with } \\
\text { distributive shock }\end{array}$ & $\begin{array}{l}\text { Clinical history suggestive of } \\
\text { cardiogenic shock with evident focus } \\
\text { of sepsis, SIRS on clinical examination }\end{array}$ \\
\hline $\begin{array}{l}\text { Hypovolemic with } \\
\text { distributive shock }\end{array}$ & $\begin{array}{l}\text { Clinical history suggestive of } \\
\text { hypovolemia with evident focus of } \\
\text { sepsis on clinical examination }\end{array}$ \\
\hline
\end{tabular}

Annexure 2: Criteria for establishing Diagnosis 2 (PoCUS alone)
Cardiogenic shock

Hypovolemic shock

Obstructive shock

Distributive shock

Cardiogenic with distributive shock

Hypovolemic with distributive shock
- Hypokinetic LV or hypokinetic LV and RV with IVC $>2 \mathrm{~cm}$ dilated and noncollapsing on PoCUS.

- Hyperkinetic LV with $>50 \%$ collapsing IVC of diameter $<2 \mathrm{~cm}$

- Only $>50 \%$ collapsing IVC of diameter $<2 \mathrm{~cm}$

- Abdominal PoCUS suggestive of free fluid with $>50 \%$ collapsing IVC of diameter $<2 \mathrm{~cm}$ with or without hyperkinetic LV

- Hypokinetic RV with multiple A lines with or without consolidation and noncollapsing IVC of diameter $>2 \mathrm{~cm}$ with or without DVT on PoCUS

- Pericardial effusion with or without hypokinetic RV and noncollapsing IVC of diameter $>2 \mathrm{~cm}$ on PoCUS

- Consolidation associated with or without air bronchogram with or without IVC collapsing on PoCUS

- Features suggestive of cardiogenic shock with evident focus of sepsis on PoCUS

- Features suggestive of hypovolemia with evident focus of sepsis on PoCUS 
Annexure 3: Criteria for establishing Diagnosis 3 (combination of data from clinical evaluation and PoCUS) —Column A + Column B

\begin{tabular}{|c|c|c|}
\hline Type of shock & Column A (clinical evaluation alone) & Column B (PoCUS alone) \\
\hline \multirow[t]{6}{*}{ Cardiogenic shock } & $\begin{array}{l}\text { Any or a combination of the below associated } \\
\text { with chest pain }\end{array}$ & $\begin{array}{l}\text { - Hypokinetic LV or hypokinetic LV and RV with } \\
\text { IVC }>2 \mathrm{~cm} \text { dilated and noncollapsing on PoCUS }\end{array}$ \\
\hline & $\begin{array}{l}\text { Syncope, pain abdomen, generalized weakness, } \\
\text { seizures, altered sensorium, vomiting, loose } \\
\text { stools }\end{array}$ & \\
\hline & $\begin{array}{l}\text { - ECG findings with ST elevation, ST depression, T } \\
\text { inversion, LBBB }\end{array}$ & \\
\hline & $\begin{array}{l}\text { - Raised JVP, pedal edema, anasarca, ascites, } \\
\text { tender hepatomegaly, hepatojugular reflex }\end{array}$ & \\
\hline & $\begin{array}{l}\text { History of progressive breathlessness, } \\
\text { paroxysmal nocturnal dyspnea, orthopnea, } \\
\text { dyspnea on exertion }\end{array}$ & \\
\hline & $\begin{array}{l}\text { Past history of cardiac, pulmonary, or renal } \\
\text { disease, diabetes, hypertension }\end{array}$ & \\
\hline \multirow[t]{4}{*}{ Hypovolemic shock } & $\begin{array}{l}\text { Any or a combination of the below associated } \\
\text { with vomiting or loose stools or both without } \\
\text { fever and normal temperature at ED evaluation }\end{array}$ & $\begin{array}{l}\text { - Hyperkinetic LV with }>50 \% \text { collapsing IVC of } \\
\text { diameter }<2 \mathrm{~cm}\end{array}$ \\
\hline & - Pain abdomen, generalized weakness & OR \\
\hline & $\begin{array}{l}\text { History of hematemesis, bleeding per rectum, } \\
\text { hemoptysis }\end{array}$ & - Only $>50 \%$ collapsing IVC of diameter $<2 \mathrm{~cm}$ OR \\
\hline & $\begin{array}{l}\text { - UPT positive with sudden onset of abdominal } \\
\text { pain }\end{array}$ & $\begin{array}{l}\text { Abdominal PoCUS suggestive of free fluid with } \\
>50 \% \text { collapsing IVC of diameter }<2 \mathrm{~cm} \text { with or } \\
\text { without hyperkinetic LV }\end{array}$ \\
\hline \multirow[t]{3}{*}{ Obstructive shock } & $\begin{array}{l}\text { - Breathlessness or chest pain with history } \\
\text { suggestive of DVT }\end{array}$ & $\begin{array}{l}\text { Hypokinetic RV with multiple A lines with or without } \\
\text { consolidation and noncollapsing IVC of diameter } \\
>2 \mathrm{~cm} \text { with or without DVT on PoCUS }\end{array}$ \\
\hline & & OR \\
\hline & & $\begin{array}{l}\text { - Pericardial effusion with or without hypokinetic RV } \\
\text { and noncollapsing IVC of diameter }>2 \mathrm{~cm} \text { on PoCUS }\end{array}$ \\
\hline \multirow[t]{6}{*}{ Distributive shock } & $\begin{array}{l}\text { Any or combination of the below with fever } \\
\text { or temperature }>100.4^{\circ} \mathrm{F} \text { at ED evaluation } \\
\text { abdomen, generalized weakness, cough, } \\
\text { seizures, vomiting, loose stools }\end{array}$ & $\begin{array}{l}\text { Consolidation associated with or without air } \\
\text { bronchogram with or without IVC collapsing on } \\
\text { PoCUS }\end{array}$ \\
\hline & - Altered sensorium, breathlessness, pain & \\
\hline & OR & \\
\hline & $\begin{array}{l}\text { - Only fever or temperature }>100.4^{\circ} \mathrm{F} \text { at ED } \\
\text { evaluation }\end{array}$ & \\
\hline & - Evident focus of sepsis on clinical examination & \\
\hline & $\begin{array}{l}\text { History suggestive of anaphylaxis, hepatic } \\
\text { insufficiency, pancreatic insufficiency, CVA, } \\
\text { nontraumatic SAH, spinal cord pathology }\end{array}$ & \\
\hline $\begin{array}{l}\text { Cardiogenic with } \\
\text { distributive shock }\end{array}$ & $\begin{array}{l}\text { Clinical history suggestive of cardiogenic shock } \\
\text { with evident focus of sepsis, SIRS on clinical } \\
\text { examination }\end{array}$ & $\begin{array}{l}\text { Features suggestive of cardiogenic shock with } \\
\text { evident focus of sepsis on PoCUS }\end{array}$ \\
\hline $\begin{array}{l}\text { Hypovolemic with } \\
\text { distributive shock }\end{array}$ & $\begin{array}{l}\text { Clinical history suggestive of hypovolemia } \\
\text { with evident focus of sepsis, SIRS on clinical } \\
\text { examination }\end{array}$ & $\begin{array}{l}\text { - Features suggestive of hypovolemia with evident } \\
\text { focus of sepsis on PoCUS }\end{array}$ \\
\hline
\end{tabular}

